\title{
PRESENZA E VALORI DEL PASSATO REMOTO IN RIASSUNTI DI OPERE LETTERARIE
}

\section{ESPRESSIONE DELLA TEMPORALITÀ TRAMITE PARADIGMI VERBALI IN TESTI SECONDARI}

In una ricerca più ampia sulle possibilità espressive della lingua italiana nel campo della temporalità, e precisamente nella fase dedicata alle caratteristiche di costruzione tipiche di testi riassuntivi e di presentazione (analizzati soprattutto sulla Enciclopedia Garzanti della letteratura [GAR] e sul Dizionario Bompiani delle opere e dei personaggi $[\mathrm{BOM}])^{1}$ si è potuta constatare una molteplicità di modi di presentazione del testo originale. Tale molteplicità risulta, da una parte, dalla maggiore o minore larghezza nell'includere nel testo secondario vari mondi testuali possibili (contenuto narrativo dell'opera originale con i propri mondi testuali, situazione storica a cui quest'opera fa riferimento, vita dell'autore e genesi dell'opera, caratteristiche letterarie e commenti valutativi, fortuna dell'opera ecc.) e, dall'atra, dall'utilizzazione di varie modalità espressive messe a disposizione dello scrivente dalla sua competeza linguistica e testuale (disposizione e ordinamento di detti mondi nel testo, procedimenti narrativi, tecniche narrative, modi di inserimento di un secondo discorso ecc.) $)^{2}$ nonché, infine, dalle scelte fatte nell'associare questi elementi. Nella nostra discussione ci limiteremo a quelle parti della presentazione che si referiscono al riassunto "puro" dell'opera originale.

\subsection{PROCEDIMENTI NARRATIVI IN RIASSUNTI}

La prima constatazione offerta dalle analisi è che, per quanto riguarda la scelta dei paradigmi verbali, nei riassunti "puri“" viene addoperato quasi esclusivamente il cosiddetto SET DI BASE: ${ }^{3}$ È il set adoperato essenzialmente per le sfere temporali dell'attualità e dell'extratemporalità; quando, invece, i suoi paradigmi vengono usati per indicare azioni appartenenti totalmente al passato (reale o immaginario) essi

- Del BOM è stato analizzato quasi l'intero primo volume e il riassunto della Divina Commedia del secondo. Analisi preliminari erano state realizzate sulla Enciclopedia Garzanti dell'arte, sull 'Atlante storico Garzanti nonchè su numerosi numeri della rivista La settimana enigmistica.

2 Della problematica tratto in L'uso delle forme verbali italiane nei riassunti di opere letterarie di contenuto narrativo: moduli espositivi tipici e loro variazioni, relazione al secondo convegno internazionale della S.I.L.F.I. (24.-27. marzo 1991 a Cambridge) prevista per la pubblicazione negli Atti.

3 Per i particolari si veda Miklič [1989], Miklič [in corso di stampa: a] e Miklič [in corso di stampa: b]. 
appaiono nella loro accezione "STORICA": il procedimento espositivo che se ne serve è stato pertanto denominato PROCEDIMENTO STORICO. Qui, nel riferimento alle azioni passate che rappresentano il FUOCO NARRATIVO - il quale man mano che il racconto prosegue si sposta avanti nel tempo-il paradigma narrativo centrale (che nel PROCEDIMENTO FONDAMENTALE è di regola il PASSATO REMOTO (PR)) è il PRESENTE STORICO (PrSt). Analogamente, anche i paradigmi della maggior parte delle azioni periferiche (anteriori, parallele e posteriori) subiscono questa trasposizione, come se interessati da una specie di "slittamento formale“. Il confronto tra il PROCEDIMENTO FONDAMENTALE e il PROCEDIMENTO STORICO potrebbe essere riassunto nel seguente grafico (che, per favorire una maggiore chiarezza, include solo l'essenziale):

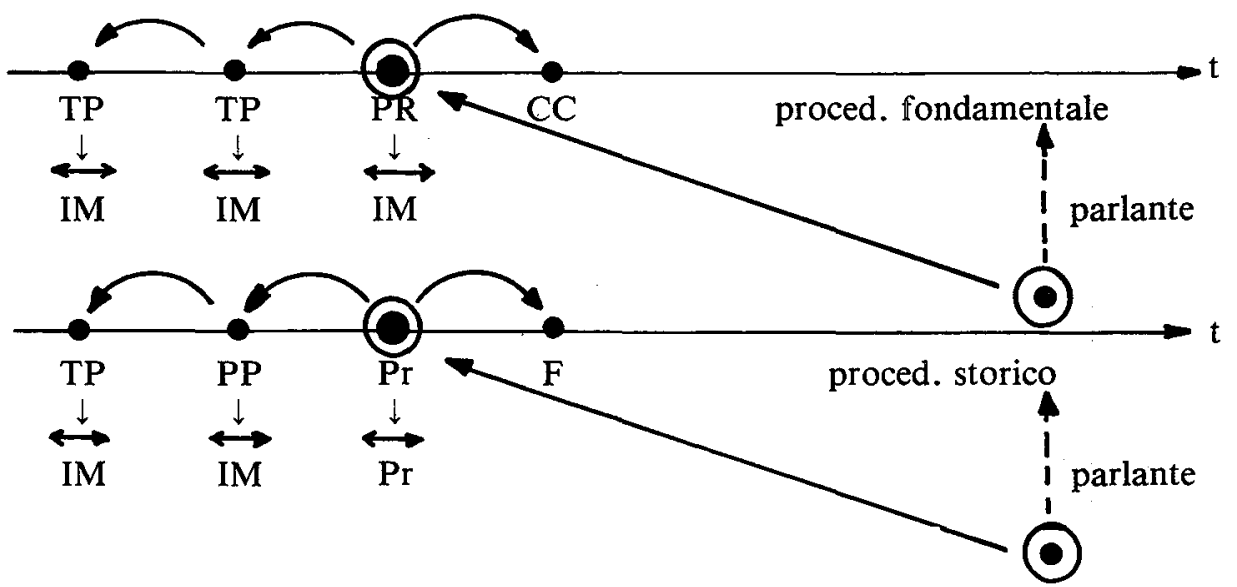

La differenza fondamentale tra $\mathrm{i}$ due procedimenti, come si può rilevare dal grafico, esiste nei paradigmi che si riferiscono ad azioni focali (PR vs Pr) e al loro immediato contorno (TP, IM e CC vs PP, Pr e F), mentre nella periferia "passata" più mediata rispetto al fuoco, le differenze sono neutralizzate. L'opposizione si estende - salvo casi specifici, ad es. nelle comparative irreali, - anche ai congiuntivi e ai condizionali. ${ }^{4}$ Illustriamo con qualche esempio:

BOM 130 La contessa di Simerose SI E SEPARATA dal marito per il disagio verecondo della prima intimità; questi, (...), per vendicarsi HA INIZIATO una relazione con un'altra donna da cui HA AVUTO un figlio. La giovane sposa CERCA allora sollievo alla sua solitudine nella corte assidua del barone De Montègre che, perdutamente innamorato di lei, $\mathbf{A C}$ CONSENTE a rimanere nei limiti di un'adorazione rispettosa. I rapporti tuttavia FINIREBBERO per trasformarsi se l'intervento del conte De Ryons non GIUNGESSE tempestivo a impedire che l'adulterio SIIA commesso. (L'amico delle donne)

4 I paradigmi verbali italiani nella loro funzione "storica" sono stati presentati al completo in altra sede (si veda la nota 2) dove vengono trattati anche i meccanismi che regolano l'uso del TRAPASSATO PROSSIMO STORICO e dell'IMPERFETTO STORICO. 
BOM 816 (...) ma subito lo SCUOTE l'alto rimprovero di Beatrice, per aver egli obliato tale bellezza quando essa ERA DIVENTATA più fulgida $e$ più pura. Maternamente, in spirito di carità, ella gli RAMMENTA le buone promesse della sua "vita nuova", e i traviamenti che non le HANNO ANNULLATE, perché esse ERANO felici disposizioni di natura a realizzare l'idea divina che AVREBBE DOVUTO, e POTRA ancora attuare. Dante CONFESSA le sue colpe (...). (La Divina Commedia)

\section{PASSATO REMOTO IN RIASSUNTI}

Stabilite le caratteristiche chiave dei due principali procedimenti narrativi, passeremo alla discussione della presenza e dei valori del paradigma PASSATO REMOTO in riassunti.

\subsection{PASSATO REMOTO NEL PROCEDIMENTO FONDAMENTALE}

Anche se raro, il procedimento fondamentale non è completamente escluso dai riassunti. In esso il PR funge da paradigma narrativo centrale, ad es.

GAR 870 Vera e propria cronaca dell'antico ponte di Višegrad, una cittadina della Bosnia, il romanzo prende le mosse dal 1516, anno in cui un ragazzino del vicino villaggio di Sokolovici, VENNE deportato dai turchi in una delle abituali retate che i dominatori facevano periodicamente. Divenuto Mehmed Ali egli FECE costruire il ponte, (...) (Il ponte sulla Drina)

Qualche volta in uno stesso riassunto l'autore passa con disinvoltura da un procedimento all'altro, ad es.

BOM 26 Il mito di Adapa (...) narra come questo figlio di Ea e sacerdote nel suo tempio di Eridu, un giorno SI TROVO in un brutto impiccio per aver spezzato le ali del vento meridionale. Egli DEVE presentarsi davanti al dio Anu per sentirsi condannare e SI RIVOLGE per consiglio a suo padre (...). (Adapa e la vita eterna)

\subsection{PASSATO REMOTO NEL PROCEDIMENTO STORICO}

Molto più interessante, e, nei riassunti analizzati, persino più frequente, è invece la presenza del PR nei testi redatti nel procedimento storico, ad es.:

BOM 155 (...) un vecchio pastore SPIEGA che Pan HA SALVATO Cloe in memoria di una Ninfa ch'egli AMO. (Amori pastorali di Dafni e Cloe).

Mentre nel procedimento fondamentale il PR designa quasi esclusivamente un'azione nel fuoco narrativo e corrisponde al PrSt nel procedimento storico, nell'ambito del procedimento storico, invece, esso si riferisce ad un'azione che è de- 
cisamente fuori del fuoco narrativo e sembra in alternanza con uno dei paradigmi storici di maggiore frequenza - il PASSATO PROSSIMO STORICO (PPSt) e il TRAPASSATO PROSSIMO STORICO (TPSt). A quanto sembra, lo scrivente si serve del PR nei punti in cui il valore degli altri due paradigmi dell' "anteriorità “ sembrano inadeguati per esprimere la relazione temporale voluta. Infatti, nel procedimento storico le azioni anteriori rispetto al fuoco narrativo vengono di norma espresse dal PPSt quando persiste una qualche RILEVANZA dell'azione anteriore anche NELLA SITUAZIONE FOCALE, mentre il TPSt sembra scelto quando l'azione designata è ANTERIORE A UN MOMENTO INTERMEDIO (sia esplicito sia implicito e deducibile dal contesto) tra il tempo dell'azione e il tempo della situazione focale - nel caso, cioè, dell'“anteriorità di II grado“, ad es.

GAR 842 Ma tornato a casa si accorge che l'ambiente in cui ERA VISSUTO SI E ADATTATO alla sua assenza: per lui, ormai non c'e più posto. (Il fu Mattia Pascal)

BOM Ma ecco giungere Anacreonte, che riferisce la triste fine di Policrate: il sovrano sconfitto E STATO CATTURATO e CROCEFISSO. Era un uomo troppo felice; gli áuguri lo AVEVANO AVVERTITO che gli dei non permettono ai mortali di essere beati, e gli AVEVANO CONSIGLIATO di procurarsi qualche dolore; e Policrate SI ERA allora PRIVATO del suo anello (...). Colpito delle parole del poeta, Agamene vuole disfarsi della statua, ma Melissa glielo impedisce. (L'anello di Policrate)

Il $\mathrm{PR}$, invece, sembra apparire proprio nei casi in cui l'azione "nel passato dei personaggi al momento del fuoco narrativo" difficilmente potrebbe essere classificata in uno dei due gruppi sopra esposti. Vediamo i seguenti esempi:

BOM 144 Il marito le DICE di non cercare delle sorelle, di non rispondere alle loro voci e di non mostrarsi a esse quando le SENTIRA piangere là sullo scoglio dove FU abbandonata, pena una grande sciagura. (Amore e Psiche)

BOM 147 (...); come Friederike ella VIVE solo del ricordo di quell'uomo, che APPARVE un giorno nella sua casa, per rapirle, quando ERA bambina ancora, il cuore. (L'amore in campagna)

BOM 117 (...) il guardacaccia, che dovrebbe rappresentare l'essere rude e semplice, capace, con la sua elementare forza fallica, di sanare e integrare la personalità della raffinata Lady, $\mathbf{E}$ in realtà un uomo che HA STUDIATO e VIAGGIATO, e che un tempo APPARTENNE a un ceto sociale non molto lontano da quello dell'amante. (L'amante di lady Chatterley) 
BOM 182 Ma Rodolfo (...) HA nel cuore una che INCONTRO fanciulla a Venezia, e ora SA sposata, ignorando a chi e dove.

(...) Questa GIUNGE fino alla stanza di Catarina quando Rodolfo ne $\mathbf{E}$ appena USCITO, e SI ERGE terribile contro la rivale. Ma, visto presso di lei un crocefisso, COMPRENDE che la donna $\mathbf{E}$ colei che un giorno SALVO sua madre da un tremendo pericolo, e ne EBBE in compenso quel dono. (Angelo, tiranno di Padova)

Come nel caso dell'uso tipico del PR nel procedimento fondamentale, dove esso ha la precisa funzione di designare azioni NETTAMENTE SGANCIATE DAL MOMENTO DI ENUNCIAZIONE (cfr. Bertinetto [1986], e in particolare p. 445), qui l'azione è indubbiamente sganciata dal centro deittico - come lo sono del resto tutte le azioni presentate nel procedimento storico-, ma in più sembra piuttosto SGANCIATA DALL" "ATTUALITA DELLA SITUAZIONE FOCALE". Quello che per l'uso abituale del PR è rappresentato dal tempo di enunciazione, per il PR "storico" sembra sia rappresentato dal fuoco della narrazione. Quest'uso, che in GAR è raro, è, invece, relativamente frequente nei riassunti d'autore in BOM. La tendenza alla regolarità nelle modalità della sua presenza non consente che sia interpretato come un casuale capriccio stilistico o un'idea stravagante di qualche singolo autore.

Grafico riasuntivo dell'opposizione PPSt vs TPSt vs PRSt

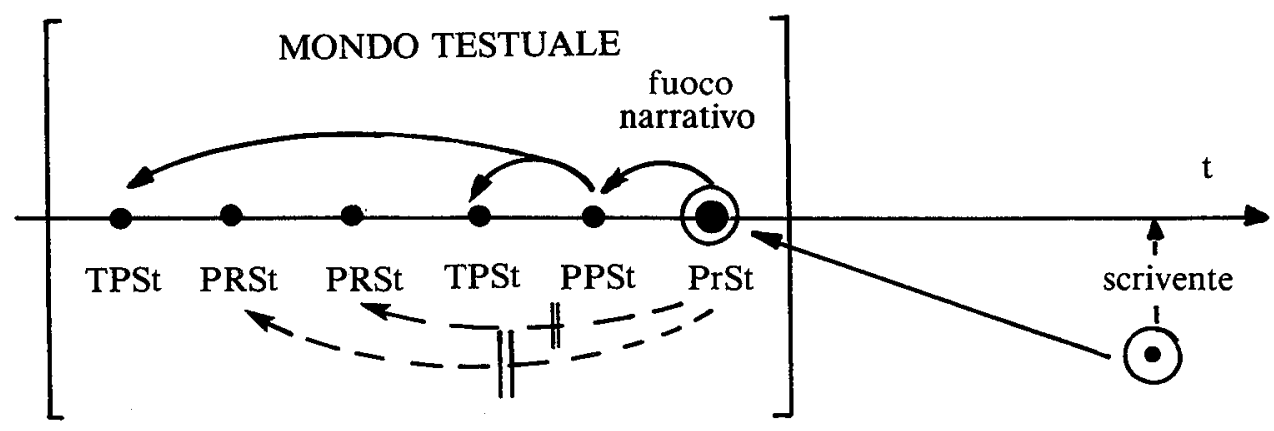

\subsection{PASSATO REMOTO IN DUE RIASSUNTI DELLA DIVINA COMMEDIA}

Un caso degno di attenzione è rappresentato poi dall'uso del PR nelle due presentazioni (GAR e BOM) della Divina Commedia. Qui si intrecciano vari mondi testuali: viaggio immaginario di Dante nei tre regni dell'Oltretomba con azioni particolari sue e di quelli che incontra, azioni extratemporali nei tre regni, avvenimenti e situazioni storiche anteriori, parallele e posteriori al viaggio, relazione di Dio con l'anima, possibilità di scelta individuale dell'uomo ecc. Anche se di dimensioni di- 
verse, i due riassunti presentano alcune caratteristiche comuni. Prima di tutto, il viaggio è raccontato tramite l'uso del procedimento storico, ${ }^{5}$ ad es.

GAR 824 Virgilio gli CONSIGLIA di seguire una strada diversa; la lupa, che HA già RESO molti infelici, non lo LASCERA passare.

GAR 827 Dante gli FA l'elogio delle virtù della sua famiglia, e Corrado gli PREDICE che questa sua opinione AVRA ragione di essere prima che PASSINO sette anni.

BOM 811 Virgilio SI OFFRE a Dante come guida. Egli lo CONDURRA giù nell'Inferno, SCALERA con lui la montagna del Purgatorio fino alla cima;

BOM 812 Ma contro l'orgoglioso Filippo Argenti, che VORREBBE risollevarsi dal fango dello Stige, Dante SCATTA con ira (...)

Una seconda caratteristica in comune riguarda l'uso del PASSATO REMOTO. I due autori se ne servono con una rilevante costanza non solo per indicare azioni che sono decisamente staccate dal fuoco narrativo, ad es.

GAR 831 (...); è Adamo, il primo uomo creato da Dio. Dante gli si inchina con reverenza e gli chiede da quanto tempo FU creato; quanto tempo RESTO nell'Eden; quale FU il peccato che SUSCITO la collera di Dio; e quale FU il suo linguaggio, domande a cui Adamo risponde.

ma anche per quelle azioni "passate" dei personaggi in base alle quali le loro anime "adesso" devono (o possono) occupare esattamente quel particolare posto nella struttura dell'Oltretomba, ad es.

GAR 829 Ella spiega che quel cielo è dedicato agli spiriti che MANCARONO ai voti.

GAR 829 I lussuriosi sono divisi in due schiere opposte che quando si incontrano si abbracciano brevemente: sono i sodomiti e i lussuriosi propriamente detti, che non SEPPERO FRENARE la natura.

5 Un'eccezione è l'inizio del viaggio nella versione BOM, dove però si pasa dal procedimento fondamentale immediatamente a quello storico che poi viene mantenuto tino alla tine:

BOM 811 Travolto dal turbine delle passioni politiche e accecato degli odi di parte, Dante, "nel mezzo del cammino di nostra vita", SI RITROVO smarrito in quella "selva selvaggia" dove ogni uomo, chiuso nella propria individualità, è natura incolta, la cui vita è simile a quella delle bestie, delle piante e delle pietre. Svegliatosi dal sonno del suo spirito, Dante TENTA subito di salire il colle della felicità irradiato dal sole; ma ne lo RICACCIANO al fondo le tre passioni che ribolono nel cuore di ogni uomo avanti l'opera della ragione (...) 
BOM 813 Qui, supini a terra, giacciono i BESTEMMIATORI di Dio; corrono precipitosamente senza posa coloro che nell'ordine dell'agire OPERARONO contro natura (sodomiti); stanno seduti, e si schermiscono dal fuoco come i cani dalle vespe, coloro che nell'ordine del fare non MISE$\mathbf{R O}$ in lucese stessi con quell'arte che s'ispira alla natura nelle sue operazioni (...)

Sembra una scelta consapevole, un mezzo stilistico: come se i due autori volessero mantenere un atteggiamento unitario nel riferisi ad azioni che appartengono ad un mondo difinitivamente separato e staccato rispetto a quello dell'Oltretomba. ${ }^{6}$ $\mathrm{E}$ interessante osservare come il traduttore della versione francese di $\mathrm{BOM}^{7}$ abbia ignorato questa regolarità del riassunto originale traducendo i PASSATI REMOTI italiani ora col PASSÉ SIMPLE ora col PASSÉ COMPOSÉ in modo del tutto arbitrario, ad es. ${ }^{8}$

BOM 812 Nei cerchi inferiori, dentro la città di Dite, sono i "maliziosi": libere volontà che violando l'ordine della ragione e della natura PASSARONO al servizio delle potenze inferiori e dell'istinto. E dopo i maliziosi, sono i bestiali: intelligenza posta al servizio di una volontà perversa, che SI FECE centro di tutto (...)

(trad. fr. 61) Dans les cercles inférieurs, dans la Cité de Dité, Dante a placé ceux qui PECHERENT par"malice": ce sont les individus doués d'une volonté libre, mais qui ONT délibérément VIOLE l'ordre naturel et rationnel, en se mettant au service des puissances passionnelles inférieurs et instinctives. Après eux, voici ceux qui ONT PECHÉ par "bestialité": leur intelligence FUT mise au service d'une volonté perverse (...)

C'è, però, anche nel testo italiano qualche mancata osservanza di questa tendenza generale: succede quando il legame con il mondo terreno si fa sentire con troppa forza, ad es.

BOM 812 Nel cerchio dei lussuriosi Dante si commuove dinanzi a Francesca, una creatura di bontà, che sospira invano alla sua pace, mentre si stringe al suo Paolo e afferma ineluttabile quell'amore che pur l'HA CONDOTTA alla morte e all'eterno affanno.

6 La scelta potrebbe, però, essere stata suggerita anche dai tanti passati remoti dell'originale. L'originale consta di parti narrative e di discorsidiretti: ma è chiaro che leazioni espresse dal PR nel raconto e quelle nel discorso diretto di un personaggio non hanno la stessa profondità temporale.

7 Dictionnaire des Oevres. Lafont-Bompiani, 1952.

$8 \mathrm{Cfr}$. anche il seguente esempio:

BOM $810 \mathrm{E}$ li, precipitando dal cielo, CADDE e sta confitto in eterno Lucifero. La terra SI RITRASSE dinanzi alla sua caduta e RICORSE in su, emergendo dalle acque dell'emisfero australe, FORMO l'isoletta del Purgatorio (...)

(trad. fr. 61) C'est là que, precipité du haut du ciel,EST TOMBÉ Lucifer; c'est là qu'il demeure pour l'éternité. Devant sa chute, la terre SE RETIRA et REMONTA en sens inverse; émergeant des eaux de l'hémisphère austral elle DONNA naissance à la petite île du Purgatoire (...) 
Anche in qualche altra situazione, poi, i due compendiatori italiani oscillano:

GAR 830 Risponde Beatrice, che se la pena della croce FU giusta se considerata inflitta alla persona umana del Cristo, essa FU ingiusta in rapporto alla sua natura divina. Prosegue dicendo che Dio HA SCELTO la morte del figlio come mezzo di redenzione del genere umano perché l'unica che comprenda insieme misericordia e giustizia.

BOM 813 È il muto dolore di Bocca degli Abati, che a Montaperti TRADÌ la parte guelfa e che a Dante non vuole per vergogna rivelare il suo nome. $\grave{E}$ il dolore del conte Ugolino, il TRADITORE della patria, che s'accanisce bestialmente sul corpo di colui che a sua volta lo HA TRADITO.

Un'altra scelta sorprendente è l'uso del PR per un'azione dentro il viaggio:

GAR 832 Mosè è accanto a Adamo (...). Di fronte a Adamo, S. Lucia che SOCCORSE Dante.

Forse si tratta solo di parallelismo formale con altri casi nel contesto, ad es.

GAR 832 (...) alla sinistra della Vergine coloro che CREDETTERO in Cristo venturo; alla destra della Vergine coloro che CREDETTERO in Cristo venuto;

L'analisi della verbalizzazione di diversi mondi testuali nei due riassunti ci porta al seguente grafico con le principali regolarità individuate:

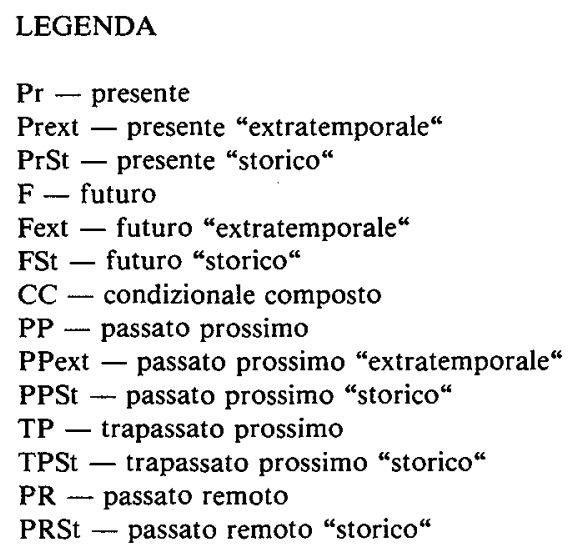




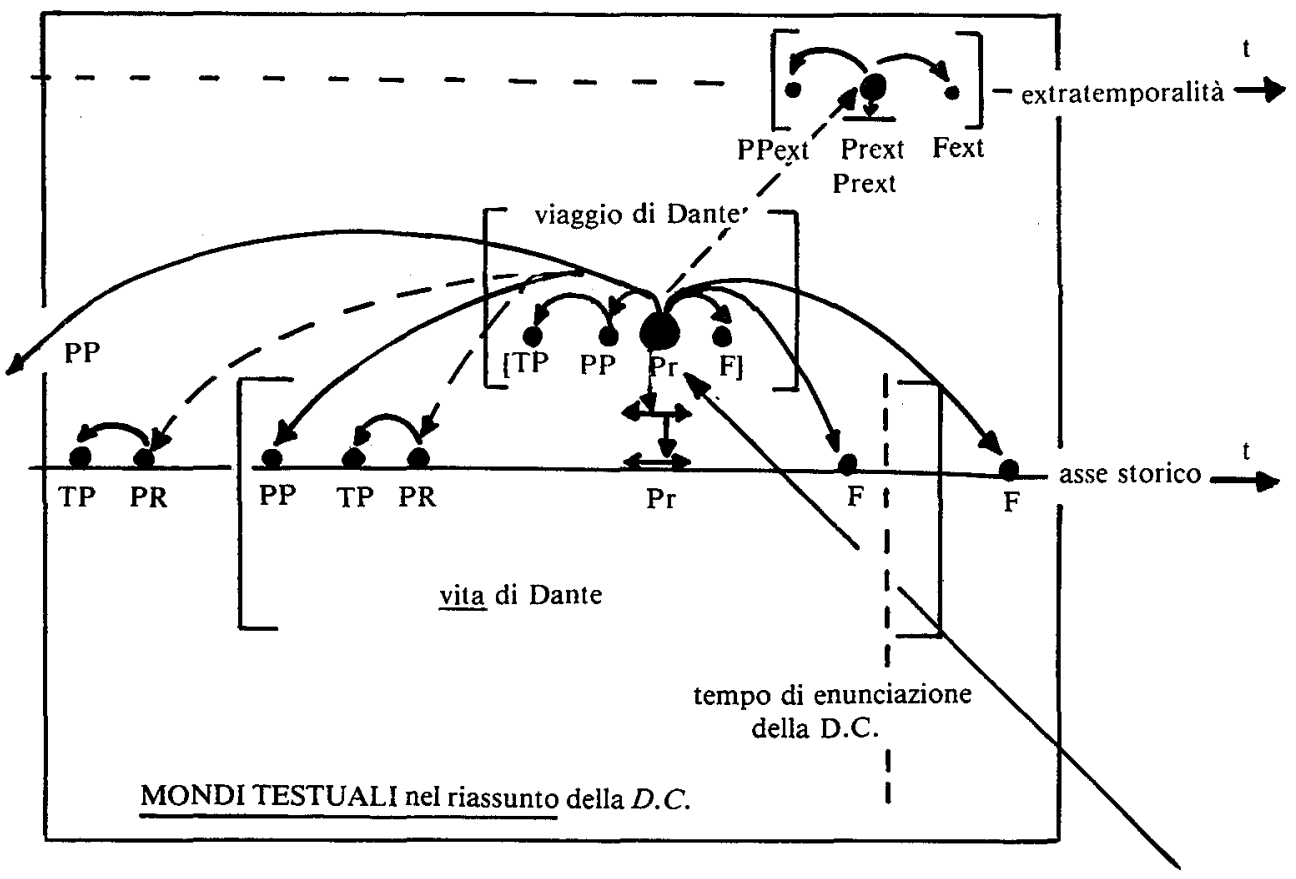

t. di enunc. del riassunto

'O

\section{CONCLUSIONE}

La analisi eseguite, come di solito accade in studi pertinenti all'ambito della creatività umana, caratterizzata dalla dialettica tra libertà e costrizione, hanno potuto enucleare soltanto alcune linee di tendenza (cfr. anche Bertinetto [1986], pp. $441 \mathrm{e}$ 445), caratteristiche di un certo tipo testuale.

\section{BIBLIOGRAFIA ESENZIALE}

Bertinetto, P.M. [1986], Tempo, aspetto e azione nel verbo italiano. Il sistema dell'indicativo. Accademia della Crusca. Firenze

Erich, V. \& Vater, H. [a cura di, 1988]. Temporalsemantik. Beiträge zur Linguistik der Zeitreferenz.

Herweg, M. [1990], Zeitaspekte. Die Bedeutung von Tempus, Aspekt und temporalen Konjunktionen. Wiesbaden

Lämmert, E. [19726], Bauformen des Erzählens. Stuttgart.

Lavinio, C. [1984], L'uso dei tempi verbali nelle fiabe orali e scritte. In: L. Coveri (cur.), Linguistica testuale. Roma. 289-306. 
Lo Cascio, V. \& Vet, C. [a cura di, 1986] Temporal Structure in Sentence and Discourse. Dodrecht.

Miklič, T. [1989] La consecutio temporum in italiano (e nelle lingue slave). Scuola Nostra 21. Rijeka-Fiume: Edit. 97-117.

Miklič, T. [in corso di stampa: a]. La Consecutio Temporum in sloveno e in italiano: alcune oservazioni. In: Atti del XXI congresso SLI (Catania 1987).

Miklič, T. [in corso di stampa: b]. La forma verbale e la sua funzione nel testo; servigi testuali del trapassato del congiuntivo. In Atti delI convegno S.I.L.F.I. (Siena 1989)

Ronconi, A. [1947] Aoristi e perfetti in Dante. Lingua Nostra, 8, 3ss; ora in: Ronconi [1958]. Interpretazioni grammaticali. Padova, 185-193.

Skubic, M. [1964]. Prispevki k poznavanju zgodovine preterita v italijanščini. Dissertazione. Ljubljana.

Skubic, M. [1978]. Sur les valeurs des temps du passé dans les langues romanes. Linguistica XVII. Ljubljana: Filozofska faklulteta. 143-159.

Skubic, M. [1986]. Passato prossimo e passato remoto nei dialetti veneti. In: Cortelazzo, M. (cur.). Guida ai dialetti veneti. VIII. Padova.

Smith, C.S. [1980]. Temporal structures in discourse. In C. Rohrer (cur.), Time, Tense and Quantifiers. Tübingen. 357-374.

Tekavčić, P. [1970]. Saggio di un'analisi del sistema verbale italiano. Lingua e Stile, $5,1-23$.

Tekavčić, P. [1972]. Grammatica storica dell'italiano. Vol. II: Morfosintassi. Bologna.

Vikner, S. 1985. Reichenbach revisited: one, two, or three temporal relations? Acta linguistica Hafniensia 19. 2. 81-98.

Weinrich, H. [1964/1971], Tempus. Besprochene und erzählte Welt. Stuttgart.

Wunderlich, D. [1970] Tempus und Zeitreferenz im Deutschen. München.

Povzetek

POJAVITVE IN FUNKCIJE PARADIGME PASSATO REMOTO

V POVZETKIH LITERARNIH DEL

Prikaz delnih rezultatov obsežnejše raziskave rabe italijanskih glagolskih paradigem se osredotoča na pojavljanje paradigme PASSATO REMOTO v povzetkih literarnih del in primerja njegovo funkcijo $v$ "historičnem" pripovednem postopku s funkcijami oblik PASSATO PROSSIMO "STORICO" in TRAPASSATO PROSSIMO "STORICO". 